\title{
Using Data in Practice: What Does It Look Like and What Does It Take?
}

Stephanie Cronen, American Institutes for Research

GeMar Neloms, American Institutes for Research

Note: This manuscript previously appeared on the ProLiteracy website. It has, however, been updated into APA 7 format.

Adult practitioners share a laudable goal—to help vulnerable adults learn and improve their lives.

To determine whether their learners are making progress or have achieved a specific outcome, most practitioners rely on data generated by a variety of formal and informal assessments. Practitioners may use these measures to adapt instruction as needed, for example, to identify learners who are struggling and need more intensive or differentiated instruction (Supovitz \& Klein, 2003; Wayman \& Stringfield, 2006). Practitioners may also find data useful for evaluating and improving instructional practices (Halverson et al., 2007; Supovitz \& Klein, 2003). Learners can use data on their own performance to inform their approach towards achieving an outcome (Hamilton et al., 2009; May \& Robinson, 2007; National Research Council, 2012). At the administrator level, program-wide data can be used to assess whether curricula or special initiatives are having the desired effect, and it can inform a change in course when needed (Kerr et al., 2006; Marsh et al., 2006).

To understand the types of outcomes data that may be useful to practitioners and how they may be used effectively, we first review existing theory and research on using outcomes data in practice. We then suggest implications for practice but also highlight gaps in this research.

\section{Guiding Frameworks}

Existing frameworks for using outcomes data in education, referred to by a variety of labels such as data-driven or data-informed decision making, are not specific to adult education. Mandinach et al., (2006) developed a commonly cited framework which conceptualizes data use as a continuum that transforms data into the actionable knowledge and understanding needed to implement effective practices. This continuum begins with collecting and analyzing data, then summarizing findings in a way that creates usable information. For example, this could be compiling math sub-scores for a program that is trying to improve instruction. This information is then synthesized to form knowledge that is useful to guide decisions and action. More specifically, a comparison of math sub-scores before and after implementing a new curriculum could be used to evaluate its success in improving learners' math outcomes. A key assumption of this model is that practitioners need both tools and knowledge- 
"data literacy"- to access and effectively analyze and make use of available data.

Marsh et al. (2006) adapted and added to this framework by making explicit the different purposes for using high-stakes assessment data and other sources. These include setting goals and assessing progress toward them, addressing individual or group needs, assessing effectiveness, or reallocating resources in response to outcomes. They also identified factors that influence data use, such as the accessibility, quality, and timeliness of data.

Means et al. (2009) conducted a large-scale implementation study to inform the development of a framework with five main actions for data use: reflecting on areas that need improvement, planning and implementing strategies to address them, collecting and analyzing assessment or other outcomes data, and reflecting on the findings to fine-tune implementation (see Figure 1). Most usefully, they detailed the critical need to focus on the conditions required for successful data use: accessible data systems, leadership focused on educational improvement and data use, tools for generating actionable data, social structures and time for analyzing and interpreting data, professional development and technical support for data interpretation, and tools for acting on data.

\section{Effects of Using Data}

Little rigorous research has been done on the effects of using data to improve learner outcomes; what does exist was conducted in K-12 settings and has shown mixed or null results. However, a small number of promising findings have emerged. For example, May and Robinson (2007) found that providing secondary students with detailed performance information led to greater persistence and improved performance in retaking the state's graduation test. An additional suggestion based on a systematic evidence review is to create a "cycle of inquiry" to help learners use formative data to reflect on their progress and become their own data-driven decision makers (Hamilton et al., 2009). ${ }^{1}$ These findings are tempered, however, by another evidence review which suggests that providing learners with timely, individualized, and ongoing qualitative feedback such as immediate identification and explanation of errors-and especially feedback that is presented within the context of a learner's goal-is more useful than sharing only test scores (National Research Council, 2012).

Several others have tested the guided use of formative or interim assessments to inform instruction and found positive effects on learning at the elementary and secondary levels 
(Carlson et al., 2011; Supovitz et al., 2018). However, impacts in one study were found only for schools with a high level of readiness to implement a data-based intervention (West et al., 2016). These findings echo the guiding frameworks described previously and reinforce the idea that there are important contextual factors to consider in using data effectively.

\section{Considerations for Practitioners}

\section{Create and Engage in a Culture of Data Use}

Creating and engaging in a culture of data use is perhaps the most all-encompassing and promising strategy to promote the effective use of data in daily practice (Means et al., 2009; Gerzon, 2015). This means that everyone who touches and is impacted by outcomes data-including learnersis supported in understanding and engaging with data because of the guidance it can provide.

Yet, creating a culture of data use in adult education programs is challenging. For example, many programs are staffed with part-time instructors, which limits their administrative, planning, and instruction time. This may contribute to low receptivity for engaging with data beyond man- dated reporting requirements. Instructors and other staff may be very receptive to integrating data into their decision making, but their access to it may be limited. Barriers like these can be addressed through supportive practices.

\section{Provide Access to and Expert Guidance on Interpreting Data}

Ongoing access and guidance on understanding and using data within a data for decision-making framework are crucial (Means, et al., 2009; Knapp et al., 2006). Strategies to accomplish this may be structural-for example, by ensuring that all instructors and paraprofessionals have access to real-time and easily understandable data and a glossary of data definitions (Jimerson \& Wayman, 2015). Tools such as data dashboards and ongoing coaching on the interpretation and application of data (see below) serve to enhance understanding and support practitioners (Murray, 2014; Means et al., 2009; Knapp et al., 2006). Additionally, it is important to ask practitioners what data would be useful to them. If request- ed data are not currently captured, staff should collaborate on ways to integrate additional data collection within an existing or future data collection system (Means et al., 2009; Knapp et al., 2006).

\section{Provide Professional Development on Data Use}

Often professional development related to data focuses on collecting and reporting information to comply with accountability mandates. Although this is necessary for all publicly funded adult education programs, it may not encourage instructor ownership of data. Helping practitioners understand, interpret, and use the data they collect to drive instructional decisions creates a meaningful context for data collection and use (Marsh et al., 2006; Murray, 2014; Shen \& Cooley, 2008). Potential strategies for accomplishing this goal include incorporating data-based scenarios and designating time in staff meetings to discuss, interpret, plan, and evaluate data results and trends. This can create opportunities to use data to inform instruction, develop or revise curriculum, and provide tailored guidance for learners.

\section{Implement Data Teams or Data Learning Communities}

Similar to the potential role of professional development, multi-disciplinary data teams or facilitated, peer-to-peer data learning com- munities can serve programs and learners by promoting a collaborative, continuous quality improvement 
approach (Gummer \& Mandinach, 2015; Jimerson \& Wayman, 2015). For example, a team comprised of a program coordinator, career navigator, literacy specialist, and ESL and ABE instructors can review learner data and collectively identify trends, outliers, challenges, and opportunities. It can hypothesize on root causes of identified issues and potential solutions or replicate promising practices for both individual learners and the program. This might include implementing activities to increase learner engagement for a specific demographic, recognizing course or service duplication, or analyzing why and how to replicate learner successes across program offerings. This process may also help identify team gaps and focus new staff recruitment on missing competencies or identify adult education partners who can help address them.

\section{Incorporate Data to Support Learner-Led Decision Making}

Instructors and other adult education staff, including career counselors, navigators, and case managers should also consider using outcomes data with learners to help them identify and periodically review progress toward the goals that first brought them to adult education (Marsh et al., 2010; Hamilton et al., 2009). This could include connecting attendance with progress towards attainment of a needed credential or certification. Or it could involve using successful high school equivalency (HSE) completion or a demonstrated increase in measurable skills gains as discussion points with individual learners for career development. Sharing data and what it means with learners may help them reflect on their persistence and highlight how to incorporate data into a résumé or use it during a job interview as an example of their potential to be successful (Murray, 2014). In this way, learners can leverage data to recognize progress and accomplishments, set new benchmarks, and identify next steps. Using data in this manner also helps professionalize the classroom by replicating the type of engagement and discussion around individual goals that may occur in the workplace.

\section{Give Data a Face and Tell a Story}

Because adult education data represents actual learners, it can be used to tell their stories as a way to increase awareness of program services and impacts. Data can inspire funders, learners, and instructors to engage with education issues and support improved learning outcomes (Gerzon, 2015; Gummer \& Mandinach, 2015). Whether it is used to show a student's successful completion of an HSE exam, progression to the next level, or fulfillment of requirements for postsecondary admission, data can be used to illustrate the journey and outcome and reinforce the value of adult education.

\section{Considerations for Research}

While existing frameworks and research offer insights into best practices for using data, there is much more to be learned. These are some of the questions that remain:

- What constitutes program readiness to use data, and how can it be supported?

- What are promising approaches to developing data literacy?

- What types of data are most useful, to whom, and for what purposes?

- How can data best be collected and made available in a format that practitioners can readily use?

- What impact does data-informed practice have on learner engagement and progress?

Additional research can inform these questions and contribute to increasing effective data use among adult education program managers, practitioners, and learners. 


\section{References}

Datnow, A., \& Hubbard, L. (2015). Teachers' use of data to inform instruction: Lessons from the past and prospects for the future. Teachers College Record, 117(4), 1-26.

Carlson, D., Borman, G. D., \& Robinson, M. (2011). A multistate district-level cluster randomized trial of the impact of data-driven reform on reading and mathematics achievement. Educational Evaluation and Policy Analysis, 33(3), 378-398.

Gerzon, N. (2015). Structuring professional learning to develop a culture of data use: Aligning knowledge from the field and research findings. Teachers College Record, 117(4), 6, 14-17.

Gummer, E. S. \& Mandinach, E. B. (2015). Building a conceptual framework for data literacy. Teachers College Record, 117(4), 14-19.

Halverson, R., Prichett, R. B., \& Watson, J. G. (2007). Formative feedback systems and the new instructional leadership. University of Wisconsin.

Hamilton, L., Halverson, R., Jackson, S., Mandinach, E., Supovitz, J., \& Wayman, J. (2009). Using student achievement data to support instructional decision making (NCEE 2009-4067). National Center for Education Evaluation and Regional Assistance, Institute of Education Sciences, U.S. Department of Education. http://ies. ed.gov/ncee/wwc/practiceguide.aspx?sid=12

Jimerson, J. B., \& Wayman, J. C. (2015). Professional learning for using data: Examining teacher needs and supports. Teachers College Record, 117(4), 1-36.

Kerr, K. A., Marsh, J. A., Ikemoto, G. S., Darilek, H., \& Barney, H. (2006). Strategies to promote data use for instructional improvement: Actions, outcomes, and lessons from three urban districts. American Journal of Education, 112(4), 496-520.

Knapp, M. S., Swinnerton, J. A., Copland, M. A. \& MonpasHuber, J. (2006). Data-informed leadership in education. University of Washington, Center for the Study of Teaching and Policy.

Mandinach, E. B., Honey, M., \& Light, D., (2006). A theoretical framework for data-driven decision making. Paper presented at the Annual Meeting of the American Educational Researchers Association, San Francisco, CA.
Marsh, J.A., Pane, J.F., \& Hamilton, L.S. (2006). Making sense of data-driven decision making in education: Evidence from recent $R A N D$ research (No. OP-170EDU). RAND.

May, H., \& Robinson, M.A. (2007). A randomized evaluation of Ohio's Personalized Assessment Reporting System (PARS). CPRE Research Reports.

Means, B., Padilla, C., DeBarger, A., \& Bakia, M. (2009). Implementing data-informed decision making in schools: Teacher access, supports and use. U.S. Department of Education, Office of Planning, Evaluation and Policy Development.

Murray, J. (2014). Critical issues facing school leaders concerning data-informed decision making. Professional Educator, 38, 14-22.

National Research Council. (2012). Improving adult literacy instruction: Options for practice and research. The National Academies Press.

Shen, J., \& Cooley, V. E. (2008). Critical issues in using data for decision making. International Journal of Leadership in Education, 11(3), 319-329.

Supovitz, J. A., Ebby, C. B., Remillard, J., \& Nathenson, R. A. (2018). Experimental impacts of the ongoing assessment project on teachers and students. CPRE Research Reports.

Supovitz, J. A., \& Klein, V. (2003). Mapping a course for improved student learning: How innovative schools systematically use student performance data to guide improvement. University of Pennsylvania, Consortium for Policy Research in Education.

Wayman, J. C., \& Stringfield, S. (2006). Technologysupported involvement of entire faculties in examination of student data for instructional improvement. American Journal of Education, 112(4), 549-571.

West, M. R., Morton, B. A., \& Herlihy, C. M. (2016). Achievement network's investing in innovation expansion: Impacts on educator practice and student achievement. Center for Education Policy Research. 